\title{
LES GUERRES EN HISPANIA ET L'ÉMERGENCE DE LA COHORTE LÉGIONNAIRE DANS L'ARMÉE ROMAINE SOUS LA RÉPUBLIQUE: UNE RÉVISION CRITIQUE
}

POR

FRANÇOIS CADIOU

\section{RÉSUMÉ - RESUMEN}

Ce travail consiste à réviser l'idée selon laquelle l'apparition et le développement de la cohorte légionnaire fut le résultat de l'expérience militaire effectuée par Rome en péninsule Ibérique dès la Seconde Guerre Punique. Cette interprétation a été essentiellement défendue par M. J. V. Bell dans un article paru en 1965, dont les principaux arguments sont discutés ici. Il en ressort que la cohorte n'était pas, aux $\mathrm{III}^{\mathrm{e}}$ et $\mathrm{II}^{\mathrm{e}}$ s. av. J.-C., une formation réservée à certaines guerres occidentales, mais un instrument tactique aussi couramment employé que le manipule, ce que tendent cependant à masquer, en dehors du domaine hispanique, les caractéristiques de notre documentation.

Este trabajo consiste en revisar la idea según la cual la aparición y el desarrollo de la cohorte legionaria fue el resultado de la experiencia militar realizada por Roma en la península ibérica desde la segunda guerra púnica. Esta interpretación la defendió en particular M. J. V. Bell en un artículo publicado en 1965 cuyos principales argumentos se rebaten aquí. De ahí surge que la cohorte, en los siglos III y II antes de Cristo, no era una formación reservada a ciertas guerras occidentales sino un instrumento táctico tan corrientemente usado como el manípulo; sin embargo, fuera del área hispánica las características de nuestra documentación tienden a enmascararlo.

\section{MOTS CLÉS - PALABRAS CLAVE}

Armée romaine. République. Hispania. Cohorte.

Ejército romano. República. Hispania. Cohorte.

\section{INTRODUCTION}

Parallèlement au processus qui transforma progressivement la cité romaine en empire méditerranéen entre le $\mathrm{III}^{\mathrm{e}}$ et le I ${ }^{\mathrm{er}}$ siècle av. J.-C., l'armée de la conquête vit également son infrastructure évoluer à l'intérieur du cadre de la militia originelle ${ }^{1}$. Une innovation particulièrement importante fut l'introduction d'une subdivision nouvelle, la cohorte (cohors), appelée à devenir à la fin de la République l'unité essentielle de la légion en remplacement du manipule (manipulus). Il s'agissait de la création d'un échelon supplémentaire, puisque le manipule avait regroupé deux centuries, tandis que la cohorte rassemblait à son tour trois manipules. La date et l'interprétation de son apparition ont été âprement discutées. On a longtemps considéré que, vers 100, la réorganisation de l'exercitus sur cette base entraîna l'effacement de la structure manipulaire, en vigueur depuis les guerres samnites de la secon-

\footnotetext{
1 Toutes les dates sont av. J.-C., sauf mention contraire.
} 
de moitié du IV ${ }^{\mathrm{e}}$ siècle. Cette substitution aurait été imposée par les insuffisances tactiques de l'organisation précédente, rendues évidentes par les défaites contre les Cimbres et aux Teutons. Elle a, par conséquent, été attribuée au train de réformes prêtées à Marius au tournant des $\mathrm{II}^{\mathrm{e}}$ et $\mathrm{I}^{\mathrm{er}}$ siècles ${ }^{2}$.

A juste titre, les difficultés soulevées par cette vision traditionnelle ont été mises en évidence très tôt. En effet, aucune source ne permet de mettre une mesure si lourde de conséquence en relation directe avec l'activité marienne, pourtant relativement bien couverte par la documentation; surtout, l'existence de la cohorte est attestée sans ambiguïté dès avant 107, par Polybe et Salluste ${ }^{3}$. Ces objections ont conduit une partie de l'historiographie à une position plus nuancée, rejetant l'idée d'une rupture brutale au début du $\mathrm{I}^{\mathrm{er}}$ siècle: de ce point de vue, le remplacement du manipule par la cohorte constituait davantage l'aboutissement d'un processus $^{4}$. Cependant, même ainsi, l'emploi de la cohorte est considéré comme une mesure demeurée très ponctuelle avant sa généralisation à l'ensemble de l'armée à la faveur du contexte nouveau des années 100, où les facteurs politiques et sociaux, en accentuant la professionnalisation des soldats et l'uniformisation de l'armée, ont joué le rôle essentiel.

Dans cette perspective, le recours à la cohorte pré-marienne, relevant de l'exception, n'est pas toujours clairement expliqué, faute d'éléments jugés suffisants. Le problème tient au fait que les mentions la concernant sont jugées trop peu fiables pour parvenir à une conclusion satisfaisante. Pourtant, le dossier relatif à la péninsule Ibérique offre de ce point de vue un éclairage intéressant. La place privilégiée qu'y tiennent les occurrences de la cohorte a été bien mise en évidence dans un article important, publié en 1965 par M. J. V. Bell ${ }^{5}$. Ce constat lui a servi à étayer une théorie séduisante associant innovation tactique et guerres en Hispania: la cohorte s'y serait substituée précocement au manipule, tactiquement et stratégiquement inadapté aux spécificités du combat contre les indigènes, avant de se diffuser progressivement à d'autres théâtres occidentaux, comme la Ligurie ou la Numidie, présentant des difficultés similaires $^{6}$. En revanche, en Orient, l'armée romaine, selon lui, aurait connu uniquement le manipule. Cette explication, souvent suivie depuis, a été mise en doute, à juste titre, mais sans avoir été précisément discutée 7 . L'objectif de ce travail est de montrer qu'elle repose sur une interprétation doublement discutable, tant sur le plan de la définition tactique de la cohorte que sur celui des conceptions guerrières propres à l'Hispania. Cela signifie qu'il faut chercher une explication autre que le particularisme hispanique aux déséquilibres de l'information livrée par les sources. A mon sens, on ne peut écarter la possibilité d'un véritable emploi tactique régulier de la cohorte dès la Seconde Guerre Punique, dont le domaine hispanique, pour des raisons que nous allons développer, conserve davantage la trace que d'autres théâtres d'opérations.

2 Marquardt, 189: p. 148-149; Steinwender, 191: p. 416-417; Parker, 1928: p. 26-28; Marín y Peña, 1956: p. 48; Delbrück, 1975: p. 414; Watson, 1969: p. 22.

3 Polybe, 11, 23, 1; Sall., Bell. Iug., 49, 6.

4 Masquelez, 1877: p. 1287; Veith, 1928: p. 376; Adcock, 1940: p. 21; Fraccaro, 1975: p. 144; Keppie, 1984: p. 63-64; Roldán, 1996: p. 48; Goldsworthy, 1996: p. 33; Goldsworthy, 2000: p. 99. En revanche, Harmand, 1967: p. 236 préfère ne pas se prononcer.

5 Bell, 1965: p. 404-419.

6 Bell, 1965: p. 413: «Warfare in Spain presented certain strategic and tactical problems which the maniple could not solve.»

7 Les conclusions de Bell ont été reprises par de nombreux travaux ultérieurs (Garlan, 1972: p. 101; Gabba, 1973: p. 1; Nicolet, 1977: p. 314; Goldsworthy, 1996: p. 35, n. 91; Goldsworthy, 2000: p. 99), non sans scepticisme toutefois (Rawson, 1971: p. 19; Wheeler, 1979: p. 306, n. 18; Keppie, 1984: p. 63); mais ces réserves sont demeurées circonscrites à de brèves remarques; Harmand, 1967: p. 237, n. 47 se contente ainsi de mentionner l'existence de la théorie. 


\section{CONCENTRATION ET DISPERSION: PROBLÈMES DE DÉFINITION DE LA COHORTE}

L'un des fondements du raisonnement de Bell est que la structure de la légion manipulaire était tactiquement et stratégiquement inadaptée aux guerres menées en péninsule Ibérique. Son argumentation est la suivante. D'une part, les intervalles ménagés entre chaque manipule dans l'ordre de bataille affaiblissaient la cohésion de l'ensemble, par conséquent vulnérable à une charge impétueuse de l'ennemi. D'autre part, le manipule divisait la légion en unités trop petites, si bien que, stratégiquement, l'armée ne pouvait pas non plus être fractionnée de manière efficace en subdivisions autonomes pour des opérations de moindre envergure. La cohorte réduisait ces faiblesses en renforçant la solidité des lignes par l'accroissement en taille de chaque unité et surtout par la disparition des intervalles les séparant. Pour Bell, la légion déployée en cohorte présentait donc l'aspect d'un front continu, contrairement à celle disposée en manipules ${ }^{8}$. D' autre part, en constituant des répliques miniatures de la légion, les cohortes devenaient désormais suceptibles d'agir de façon autonome'

La difficulté tient essentiellement au premier de ces deux arguments. Sur le plan tactique, il ne me semble pas en effet que la cohorte puisse être aussi radicalement opposée au manipule. En principe, la cohors regroupait trois manipuli de chacune des lignes composant la légion (hastati, principes, triarii) ${ }^{10}$. Peut-être n'en avait-il pas toujours exactement été ainsi puisque Polybe ne précise pas quels types de manipules sont rassemblés dans la cohorte qu'il mentionne pour l'année 206: on ne peut rejeter l'idée qu'ils pouvaient alors appartenir tous à une même ligne. Quoi qu'il en soit, l'important est de relever que, dès cette époque, la cohorte était bien définie, de l'aveu même de l'historien grec, comme le triple du manipule, c'est-à-dire de l'unité de base de la légion ${ }^{11}$. Par conséquent, on ne peut nier que la création de cet échelon supplémentaire avait marqué une volonté de former des unités de taille supérieure: la légion entièrement organisée ainsi ne comptait plus que dix subdivisions au lieu de trente $^{12}$. En ce sens, il s'agissait bien d'une concentration. Mais il n'est pas possible d'en déduire que ces regroupements signifiaient pour autant la constitution d'un front plus massif, uni et rectiligne, comme le pense Bell qui associe pratiquement la cohorte à un retour à la phalange originelle.

L'introduction de la nouvelle subdivision ne peut en effet être comprise comme l'abandon d'une dispersion tactique jugée insatisfaisante. Certes, la légion manipulaire avait bien introduit une certaine dislocation dans la masse compacte de la phalange. Elle était désormais rangée en bataille sur trois lignes au lieu d'une et chaque manipule se trouvait séparé de son voisin par un intervalle selon une disposition en échiquier qu'on a pris l'habitude de désigner par le terme de quincunx ${ }^{13}$. Mais cette formation déconcentrée représentait précisément ce qui faisait la force et l'originalité de la légion. Les intervalles permettaient de maintenir une meilleure cohésion de l'ensemble au cours des déplacements sur le terrain, tandis que le triplement de la ligne de combat maintenait utilement une partie des troupes en réserve, disponible pour intervenir en cas de besoin. Pour cette raison, les intervalles n'étaient sans doute pas comblés au moment de l'assaut, contrairement à ce que l'on a souvent cru: ils pouvaient servir à renforcer le front avec des troupes fraîches, au cours des nombreux temps

8 Bell, 1965: p. 409: «In a word, to the Romans, tactically the maniple implied dispersion, and the cohort concentration.»

9 Bell, 1965: p. 411.

10 C'est du moins ce que suggèrent les titres des centurions au $\mathrm{I}^{\mathrm{er}}$ s. cf. Keppie, 1984: p. 174.

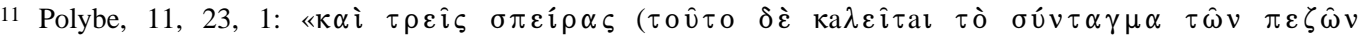
$\pi \alpha \rho \grave{\alpha}$ 'P $\omega \mu \alpha$ íoı $\varsigma$ кó $\rho \tau \imath \varsigma.) . »$

12 Gell., NA, 16, 4, 6.

13 Cette disposition particulière est restituée d'après Liv., 8, 8, 9-13. Mais le terme quincunx lui-même a été forgé par les modernes. 
morts qui caractérisaient le combat dans l'Antiquité14. Pendant l'assaut, la faible profondeur des manipules de première ligne était compensée par l'alternative fournie par les deux suivantes: si les hastati se révélaient incapables de résister à la pression de l'adversaire, celui-ci se retrouvait immédiatement confronté aux principes, puis, dans les cas désespérés, aux triarii, ultime rempart avant la déroute Ainsi, l'ordre ouvert de la légion manipulaire constituait un dispositif parfaitement adapté pour absorber un choc frontal avec une ligne ennemie.

De fait, ce principe de la triplex acies, caractéristique de la légion manipulaire, est bel et bien demeuré en vigueur dans sa version en cohorte, jusqu'à la fin de la République et audelà ${ }^{15}$. Rien ne permet de penser qu'il a donné lieu à une interprétation différente à cette époque $^{16}$. Bell conclut pourtant à un front continu des cohortes en raison des mesures prises par Scipion à Zama en 202, telles que nous les rapportent Tite-Live et Frontin ${ }^{17}$. Mais c'est oublier que Polybe se contente d'affirmer que Scipion renonça simplement au quincunx pour aligner ses manipules les uns derrière les autres ${ }^{18}$. Peut-être le récit des premiers, qui écrivent à une époque très postérieure aux faits, a-t-il été influencé par le fait qu'à l'époque césarienne, les trois manipules composant la cohorte étaient formés en bataille côte-à-côte et non l'un derrière l'autre, comme auparavant. Alors même, cette disposition sur un seul front devait inévitablement comporter également des intervalles ${ }^{19}$. L'interprétation que Bell donne d'un passage de Frontin relatif à la bataille de Pydna ne saurait en aucun cas servir à réfuter cette idée. L'auteur flavien écrit en effet: «triplicem aciem cuneis instruxit, inter quos uelites subinde emisit.» ${ }^{20}$. Bell croit que Frontin, habitué à un alignement ininterrompu de cohortes, se méprend sur la présence d'intervalles dans la légion manipulaire et les présente à tort comme un stratagème ${ }^{21}$. Il est pourtant clair qu'il décrit simplement une tentative du général romain pour donner aux différents segments de sa ligne de bataille davantage de capacité perforante, face à la redoutable phalange de Persée, en adoptant une formation en plusieurs cunei $^{22}$. Bell considère enfin l'expression «manipulos laxare», employée à plusieurs reprises par TiteLive, César ou Frontin, comme une preuve supplémentaire d'une densité supérieure de la ligne formée en cohorte, dont les manipules ne présenteraient donc plus de discontinuité entre eux ${ }^{23}$. Selon moi, elle montre surtout l'élasticité des intervalles dont les généraux romains

14 Cette question des intervalles a été l'une des plus débattues entre les spécialistes de l'armée romaine. Beaucoup considèrent en effet improbable, voire impensable, leur maintien durant la phase du combat (Delbrück, 1975: p. 273; Connolly, 1981: p. 141; Connolly, 1989: p. 162; Keppie, 1984: p. 39). A la suite des objections de Veith, 1928: p. 359361 et d'Adcock, 1940: p. 9-10, la position inverse tend actuellement à l'emporter, comme en témoignent les travaux récents de Goldsworthy, 1996: p. 208 et Sabin, 2000: p. 16.

15 Marquardt, 1891: p. 149 pensait que les dix cohortes se déployaient sur une seule ligne; mais les sources montrent le contraire, puisqu'elles n'attestent qu'un seul exemple de ligne unique, dans un contexte particulier (Bell. Afr., 13). Sur cette question, voir Veith, 1928: p. 428-429; Fraccaro, 1975: p. 147-148; Goldsworthy, 1996: p. 137.

16 Wheeler, 1979: p. 307, a bien montré qu'à l'époque républicaine, la formation traditionnelle en phalange compacte, à laquelle il faut sans doute rattacher la testudo, n'intervenait qu'en cas de circonstances exceptionnelles.

17 Liv., 30, 33, 1: «Non confertas autem cohortes ante sua quamque signa instruebat sed manipulos aliquantum inter se distantes.»; Front., Strat., 2, 3, 6: «Nec continuas construxit cohortes, sed manipulis inter se distantibus spatium dedit.» Sur l'interprétation de ces passages, voir Bell, 1965: p. 409.

18 Polybe, 15, 9, 7.

19 Veith, 1928: p. 378.

20 Front., Strat., 2, 3, 20.

21 Bell, 1965: p. 408: «A manipular legion would of course look like a series of wedges when seen from in front; what is significant is that Frontinus thought of this as a stratagem, something unusual and out of the way.» On voit mal cependant comment l'erreur de Frontin, écrivant plusieurs siècles après les faits, pourrait provenir d'une illusion d'optique. D'autre part, Bell oublie, qu'en raison de la disposition en quincunx, la légion vue de face paraissait, au contraire, présenter un front continu.

22 C'est seulement dans un second temps, devant l'échec de ce premier déploiement, que Paul-Emile se résolut à employer alors le stratagème qui fait l'objet de l'intérêt de Frontin: un simulacre de fuite.

23 Bell, 1965: p. 409. Pour ce faire, il s'appuie essentiellement sur la lecture de Caes., BG, 2, 25. Mais le texte ne dit rien de tel: le déroulement du récit, relatif à un combat sur la Sambre, implique que les légionnaires avaient été sur- 
pouvaient faire varier l'ampleur en fonction des circonstances: c'est ainsi qu'il faut comprendre l'épisode de 211, relatif à la péninsule Ibérique, où Marcius augmenta de la sorte les espaces entre les manipules afin de permettre à l'ennemi encerclé de pouvoir fuir et, ce faisant, d'affaiblir sa cohésion ${ }^{24}$. La persistance de cette pratique jusqu'à l'époque de César confirme que les intervalles n'étaient pas considérés comme une faiblesse, mais bien comme un élément à part entière du dispositif de bataille, dont le général pouvait tirer parti à son avantage.

Il est donc trop réducteur de faire de la cohorte l'antithèse tactique du manipule. Au contraire, la première prolongeait, en la complexifiant, l'originalité profonde du second. Pour cette raison, le manipule est resté jusqu'au Haut-Empire une division de base de la légion. Le regroupement en cohorte, en introduisant un maillon supplémentaire dans la chaîne de commandement, simplifiait seulement au général la direction de ses unités et permettait ainsi une coordination plus subtile des manœuvres ${ }^{25}$. Au I ${ }^{\mathrm{er}}$ siècle, l'uniformisation accrue entre chaque unité ne fit au fond que multiplier les combinaisons tactiques envisageables: alors que, jusqu'à présent, les manipules de triarii étaient armés différemment et comptaient moitié moins d'hommes que ceux des deux autres lignes, toutes les cohortes étaient désormais identiques et interchangeables, échappant ainsi à l'ancienne spécialisation tactique des trois lignes de la légion manipulaire ${ }^{26}$. Envisager le recours à la cohorte comme un retour à la rigidité de la phalange revient à se méprendre sur la signification profonde de cette évolution qui introduisait au contraire un degré accru de sophistication. Sa diffusion témoignait avant tout d'une diversification croissante des solutions tactiques à la disposition des légions. Cette inflexion se faisait par conséquent dans le même esprit de flexibilité que sur le plan stratégique, où, comme l'affirme Bell à juste titre, l'autonomie renforcée de la cohorte lui permettait effectivement d'agir isolément, sans craindre une vulnérabilité trop grande.

\section{LES LIMITES DE LA SPÉCIFICITÉ DES GUERRES EN HISPANIA}

Une cohorte alliant concentration tactique et flexibilité stratégique, telle que la conçoit Bell, n'existe pas dans la documentation. Il s'agit à mon sens d'une première objection sérieuse à l'idée que c'était précisément cette double caractéristique qui avait fait de la cohorte le type d'unité idéal pour les guerres menées en péninsule Ibérique. En outre, celles-ci ne présentent pas l'aspect qui, dans cette perspective, aurait requis une telle innovation. Bell justifie en effet le besoin d'une concentration tactique par l'impétuosité des charges des armées indigènes, plus redoutables même que celles des Gaulois ou des Cimbres, tandis que la dispersion stratégique aurait été parallèlement imposée par une guérilla perpétuelle. Mais cette affirmation, censée expliquer l'abandon précoce du manipule, ne correspond en fait à rien de solidement établi pour la péninsule Ibérique.

Il est certain, on l'a rappelé précédemment, que la flexibilité introduite par la cohorte dans l'ordre de bataille de l'armée romaine facilitait aux généraux le fractionnement éventuel de leurs forces afin de conduire des opérations de moindre envergure dans des régions où

pris par l'attaque des ennemis. En 2, 21-22, César précise même qu'ils n'avaient pu former l'ordre de bataille avant d'être durement pressés par l'adversaire. L'ordre donné consistait donc avant tout à désserrer les rangs anormalement imbriqués les uns dans les autres. En ce sens, le passage offre donc plutôt un argument en faveur d'une pratique ordinaire où les intervalles entre les manipules composant les cohortes étaient jugés indispensables à l'efficacité des combattants.

24 Front., Strat., 2, 6, 2: «laxatis manipulis et concesso fugae spatio dissipatos sine periculo suorum trucidauit.» Je ne crois donc pas qu'on puisse suivre Bell lorsqu'il affirme qu'un tel ordre n'a de sens que dans une formation en cohorte. Pour cette raison, il me semble difficile d'utiliser ce texte, comme il le fait, comme une preuve supplémentaire de l'existence de la cohorte en péninsule Ibérique dès 211.

25 Ce point a été bien mis en évidence par Goldsworthy, 1996: p. 34

26 Sur les différentes possibilités de déployer la légion en cohorte, voir Delbrück, 1975: p. 414; Fraccaro, 1975: p. 148-149; Goldsworthy, 2000: p. 99. 
l'émiettement politique de l'adversaire est une réalité bien attestée par notre documentation. Assurément, Rome ne s'était pas trouvé confrontée en péninsule Ibérique à une puissance politique centralisatrice comparable aux grandes monarchies hellénistiques qui lui firent face en Méditerranée orientale. Il faut toutefois se garder d'assimiler cette absence d'unité à une stratégie de guérilla qui en serait le corollaire naturel.

Contrairement à une idée répandue, la guérilla n'était pas le recours le plus évident de protagonistes dont les moyens humains et matériels apparaissent pourtant aux historiens comme incapables d'être rapportés à ceux du conquérant: elle réclame en effet également une conception guerrière qui ne semble pas avoir dominé parmi les peuples péninsulaires ${ }^{27}$. Il me semble très important à ce titre de relever que, dans son raisonnement, Bell ne démontre pas l'existence d'une guérilla ibérique, mais la considère comme un fait acquis, presque un trait de civilisation ${ }^{28}$. Or, les travaux les plus récents sur la guerre antique montrent que, d'une manière générale, les structures politiques, économiques et sociales des sociétés barbares, loin de favoriser une guerre de coup de main prolongée, incitaient au contraire les armées indigènes à rechercher une décision rapide en bataille rangée, selon un schéma très proche de ce que l'on connaît pour le monde grec classique et hellénistique ${ }^{29}$. Les sources, littéraires et archéologiques, montrent ainsi que l'armement et le déroulement des combats privilégiaient effectivement, dans le monde ibère des $\mathrm{III}^{\mathrm{e}}$ et $\mathrm{II}^{\mathrm{e}}$ siècles, un combat en formation moins éloigné qu'on a pu le dire de celui pratiqué par les Romains eux-mêmes ${ }^{30}$. Les quelques textes habituellement allégués pour soutenir l'hypothèse d'une guérilla indigène concernent uniquement les populations de l'intérieur, Celtibères et Lusitaniens ${ }^{31}$. Surtout, ils proviennent d'ouvrages à caractère géographique ou ethnographique dont l'information doit être précisément resituée dans cette perspective particulière, sous peine de contresens. De fait, les récits de la pénétration romaine dans ces régions contredisent les généralités tirées de ces seuls documents ${ }^{32}$. Même s'il faut sans doute distinguer dans le détail des pratiques stratégiques et tactiques variables selon les peuples confrontés à Rome, force est de reconnaître que le recours à la guérilla ne se déduit pas de la documentation disponible. Plus probablement, la dispersion stratégique permise par la cohorte correspondait davantage aux besoins nés des conceptions romaines elles-mêmes en matière de conduite des opérations militaires, où la nécessité d'une réserve, l'élaboration de manœuvres complexes ainsi que l'habitude de détachements à des fins diverses avaient contribué à introduire sans cesse plus de souplesse dans l'organisation de la légion ${ }^{33}$.

Bien que la bataille rangée fut vraisemblablement beaucoup plus répandue que ne le suppose Bell, rien n'autorise cependant à supposer qu'elle posa en péninsule Ibérique des difficultés spécifiques, réclamant la création d'une unité tactique plus compacte que le manipule. Les textes avancés par lui à cette fin sont trop disparates et ne permettent pas d'aboutir à une

27 Ce point a été très justement rappelé par Quesada, 1996: p. 142.

28 Bell, 1965: p. 411: «in Spain, the home of the word guerilla, pitched battle are rarely decisive.» Les seuls arguments avancés ensuite en ce sens sont la «Spanish incapacity for unity» et le passage de Bell. Hisp., 8, 3-5 relatif à la qualité défensive des nombreux hameaux fortifiés (turris) d'Hispanie méridionale. Ces deux éléments ne nous disent pourtant rien des traditions guerrières indigènes. L'image que l'auteur se fait de celles-ci s'inscrit par conséquent avant tout dans une tendance qui, depuis la fin du XIXe s., plaque sur le monde ibérique antique, dans une optique nationaliste, l'esprit de résistance des guérillas hispaniques de l'époque moderne, en particulier lors de l'épisode napoléonien. Sur cette dimension de la notion, voir les remarques judicieuses de Quesada, 1997a: p. 654, n. 2.

29 Goldsworthy, 1996: p. 78.

30 Quesada, 1997a: p. 615.

31 Strab., 3, 3, 6 et Diod., 5, 34, 4-7.

32 La bataille rangée fut le mode normal d'affrontement, aussi bien contre les Celtibères (Liv., 35, 7, 8; 35, 22, 8; 39, 21, 3; 40, 30, 6; 40, 50, 2-3) que contre les Lusitaniens (Liv., 35, 1, 5-11; 37, 46, 7; 37, 57, 5; 39, 21, 2; App., Ib., $56 ; 57 ; 58 ; 67)$.

33 Sur l'association dans les sources de la cohorte légionnaire à ces aspects de la guerre romaine, voir infra, p. 157. 
telle conclusion. Ainsi, un passage fameux de César (Caes., $B C, 1,44,1-2)$ est-il invoqué pour démontrer l'efficacité du concursus indigène face à une armée romaine. Mais il s'agit en fait d'une petite digression, relative à l'acculturation des troupes afraniennes en 49 , qui ne doit pas être surinterprétée. Dans le récit, elle fait en effet suite à la mention d'un repli d'un détachement césarien, incapable de s'emparer d'une légère hauteur devant Ilerda ${ }^{34}$. L'objectif de César était, de l'aveu même de celui-ci, particulièrement important: il s'agissait de couper la ligne de ravitaillement ennemie en prenant position sur un point situé à michemin entre la ville et le camp pompéien ${ }^{35}$. La manoeuvre n'en demeurait cependant pas moins fort téméraire, puisque la hauteur se trouvait à trop grande proximité de l'ennemi, au milieu d'une petite plaine assez étroite que César évalue à trois cent pas seulement. Cette prise de risque exagérée explique l'échec cuisant de la tentative ${ }^{36}$. César cherche alors à la masquer en insistant sur la désorientation momentanée de ses troupes, face à une technique inhabituelle des soldats pompéiens qu'il profite, par la même occasion, pour assimiler insidieusement à des Barbares. Le procédé est bien connu et consiste, par un subtil processus de déformation, à minimiser les erreurs stratégiques de César par la mise en valeur d'éléments secondaires ${ }^{37}$. Il est frappant, si l'adoption d'une tactique indigène était véritablement l'élément qui avait donné aux pompéiens l'avantage sur leurs adversaires, qu'aucun autre passage du Bellum Ciuile ne fasse écho d'une semblable supériorité, pourtant propre à mettre en relief le mérite de la victoire césarienne ${ }^{38}$. Cela suggère que, plus vraisemblablement, la remarque de César remplit bien, à l'intérieur du récit, la fonction tout à fait précise et ponctuelle que je viens de commenter. La mettre alors en relation, comme le fait Bell, avec ce que Tite-Live nous dit de l'efficacité du cuneus celtibère pour les années 185 et 182 n'est pas de bonne méthode, d'autant plus qu'il s'agit d'un contexte chronologique et ethnique fort différent ${ }^{39}$. D'autre part, la formation adoptée à ces occasions par les Celtibères ne caractérise pas particulièrement une technique de combat indigène, mais appartient au registre général de la bataille rangée: il s'agit de l'une des dispositions que peut adopter une armée formée en bataille afin de percer la ligne adverse ${ }^{40}$. Rien dans le texte de Tite-Live ne permet de penser à une originalité de la version celtibère ${ }^{41}$. Ces passages suggèrent surtout que les affrontements entre Celtibères et Romains se sont en règle générale déroulés selon un schéma relativement classique, dont les armées ibériques maîtrisaient les données fondamentales. L'interprétation de la bataille rangée indigène proposée par Bell présente finalement le même défaut souligné précédemment à propos de la guérilla. Les quelques exemples tirés des sources servent seulement à illustrer des considérations générales fondées sur des schémas supposés intempo-

34 Caes., $B C, 1,43,5$.

35 Caes., $B C, 1,43,2$.

36 Caes., $B C, 1,43,4$ reconnaît ainsi que les troupes d'Afranius étaient arrivées sur place «breuiore itinere».

37 Rambaud, 1966, notamment p. 204-208.

38 Face à ce silence de César, il me semble par conséquent difficile de soutenir, comme Bell, 1965: p. 411, que l'anecdote d'Ilerda traduit une efficacité particulière de la tactique indigène: «this is implied by Caesar where he ascribes the defeat of some of his finest troops to the adoption by the Afranians of Spanish methods of warfare.» Au contraire, Caes., BC, 1, 47 précise que les Afraniens avaient la réputation d'être des soldats moins capables («cum esse omnium iudicio inferiores viderentur»).

39 Liv., 39, 31, 3 et 40, 40, 2. Le rapprochement avec le passage de César est pourtant considéré par Bell, 1965: p. 411 comme un élément de preuve.

40 Le cuneus est ainsi décrit par Veget., Epit. Rei Milit., 3, 19.

41 Bell, 1965: p. 411 estime que le cuneus celtibère possédait une force de pénétration d'autant plus redoutable qu'il s'appuyait sur l'emploi d'une épée de qualité très supérieure à celle des Gaulois et des Romains. L'argument n'est guère probant. Il faudrait d'abord être certain que l'efficacité d'une formation de ce type reposait sur un corps-à-corps à l'épée. Ce n'est pas ce que suggère le texte de Végèce cité à la note précédente, selon lequel cette disposition permettait de rompre la ligne ennemie: quia a pluribus in unum locum tela mittuntur. Quoi qu'il en soit, il faut également se garder de faire de l'arme de poing un atout décisif en faveur des indigènes: on sait que les Romains disposaient sans doute, dès la fin du III ${ }^{\mathrm{e}}$ s., d'une épée similaire à celle des Celtibères. Sur ce point, voir en dernier lieu Quesada, 1997b: p. 251-270. 
rels ${ }^{42}$. Le cas de l'Hispania montre que cette vision stéréotypée finit par déformer l'analyse des évolutions de l'armée romaine elle-même: c'est ce qui apparaît clairement en ce qui concerne l'apparition de la cohorte légionnaire.

Les données de l'archéologie, relativement abondantes pour l'Hispania dans le domaine militaire, appuient ce constat. Les camps républicains autour de Numance, les seuls à nous livrer des éléments de leurs structures internes, ne permettent pas en effet de vérifier sur le terrain une hypothétique transition du manipule à la cohorte. Schulten croyait en reconnaître la trace dans les baraques de Peña Redonda et de Renieblas V, dans la mesure où elles présentent une disposition différente de celles de Renieblas III, rattaché aux guerres celtibères, dans lequel il voyait l'illustration concrète du camp manipulaire, tel que Polybe le décrit dans son livre VI. Peña Redonda, daté par lui du siège de Numance, aurait représenté une première étape, assimilable à une proto-cohorte, tandis que Renieblas V, qu'il attribuait à Titurius, un légat de Pompée en 75 , aurait reflété alors la castramétation postérieure à la généralisation de la cohorte ${ }^{43}$. La datation de ce dernier site repose toutefois sur des bases très fragiles, si bien qu'on a pu proposer, sur des critères numismatiques, d'y voir plutôt un camp contemporain de Peña Redonda ${ }^{44}$. Il semble cependant préférable de ne pas retenir une chronologie trop précise et de conserver prudemment une intervalle large entre l'extrême fin du $\mathrm{II}^{\mathrm{e}}$ siècle et les guerres sertoriennes ${ }^{45}$. Quoi qu'il en soit, la pauvreté des structures mises au jour, y compris dans la partie nord-est du site, la mieux connue, exclut de pouvoir accepter la restitution de son plan telle que l'a proposée Schulten: très proche du modèle impérial, elle est surtout le fruit de la conception préconçue que le savant allemand avait de l'aspect d'un camp romain postérieur à la Guerre Sociale, mais ne correspond à rien sur le terrain ${ }^{46}$. Le camp $\mathrm{V}$ de Renieblas n'est, par conséquent, d'aucune utilité pour démontrer que l'organisation interne des baraquements des camps républicains d'Hispania avait pu précocement refléter une nouvelle formation tactique.

Le cas de Peña Redonda est plus problématique. L'aspect des structures situées au sud du praetorium, en raison de leur forme allongée, rappelle à première vue les hemistrigia qu'on connaît pour le Haut-Empire ${ }^{47}$. Mais il paraît difficile de les inscrire dans un ensemble cohérent, susceptible de prouver que la circonvallation de Numance abritait une légion déjà organisée en cohorte, comme on le veut parfois en rapprochant Peña Redonda de deux des autres sites qui la composent (Castillejo III et Molino) ${ }^{48}$. Tout comme pour Renieblas V, le plan restitué par Schulten pour Castillejo III est suspect et sa précision tient seulement à la conviction que le site avait abrité le quartier général de Scipion $^{49}$. La superposition de différentes phases complique en outre considérablement l'interprétation des vestiges dont la dispari-

42 Bell, 1965: p. 410: la difficulté supposée des Romains face aux Barbares d'Extrême-Occident relevant à ses yeux du cas où une armée moderne est mise en déroute par un adversaire moins avancé, il se contente d'en expliquer longuement le principe au moyen de l'exemple de la défaite des troupes anglaises face aux Ecossais à Prestonpans en 1745 et d'une citation tirée de l'Art de la Guerre de Sun-Tzu (4, 27).

43 Peña Redonda: Schulten, 1927: p. 135; Renieblas V: Schulten, 1929: p. 128 et 161.

44 Hildebrandt, 1979: p. 26, suivi par Dobson, 1996: p. 39.

45 Pamment, 1996: p. 27.

46 Le plan schulténien est unanimement critiqué par Pamment, 1996: p. 119 et Dobson, 1996: p. 198.

47 Schulten, 1927: p. 163-164; Pamment, 1996: p. 99; Dobson, 1996: p. 276.

48 C'est l'hypothèse de Dobson, 1996: p. 67: selon lui, les vestiges de Peña Redonda, de Castillejo III et de Molino attestent un type de baraques où chacun des trois manipules formant la cohorte est placé derrière le précédent. Il y ajoute ce que Schulten considérait comme l'annexe du camp III de Renieblas et qu'il propose, pour sa part, d'interpréter comme un camp supplémentaire, datable du siège de Numance, qu'il appelle camp VI. Mais, bien que le lien de ce dernier avec le camp III ne repose effectivement sur aucune base solide, il n'en va pas différemment de son rattachement aux événements de 133. Le seul argument de Dobson est, en effet, une similitude supposée avec le plan des sites de Numance.

49 Voir les critiques méthodologiques de Pamment, 1996: p. 85-90. 
tion, à l'heure actuelle, interdit malheureusement tout réexamen de première main ${ }^{50}$. De la même façon, la structure interne de Molino, plus mal connue encore, se prête aux suppositions les plus variées ${ }^{51}$. L'amplitude de la marge d'incertitude ne saurait donc permettre d'utiliser ces deux exemples pour fonder solidement une hypothèse. D'une manière générale, il faut ainsi admettre que les vestiges sont beaucoup trop fragmentaires pour parvenir à des conclusions fermes quant au plan des camps numantins, même dans le cas de Peña Redonda, où l'état de conservation est le meilleur. Car, en supposant que ce soit possible, établir la forme et l'organisation de constructions ne suffit pas. Il faudrait être capable de déterminer en outre les catégories de troupes qu'elles ont chacune abritées, ce qui est loin d'être le cas, malgré les affirmations de Schulten. Surtout, il est très loin d'être certain que les camps de la circonvallation de Numance soient tout à fait représentatifs de la castramétation républicaine. Insérés dans une enceinte, ils appartenaient en effet plutôt à un ensemble de forts entre lesquels les troupes étaient réparties selon une logique que nous ignorons ${ }^{52}$. Des critères circonstanciels, notamment la nécessité d'adapter leur organisation aux contraintes de la topographie, intervenaient également pour modifier la disposition ordinaire des bâtiments ${ }^{53}$.

Pas plus que l'opposition tactique de la cohorte et du manipule, l'exception hispanique n'apparaît donc clairement dans les sources. Si la manière de combattre des Barbares, et notamment des Celtibères, avait contribué à imposer le recours exclusif à une nouvelle formule tactique, il est curieux que les récits liviens pour 185 et 182 ne fassent aucune référence à la cohorte comme parade au cuneus, alors même qu'il s'agit là de deux des descriptions de bataille parmi les plus détaillées que nous possédons pour l'Hispania de cette époque et que nous connaissons par Polybe le recours à la cohorte en péninsule Ibérique depuis au moins 206. A l'inverse, il n'apparaît nullement gênant à Bell que cette mention polybienne, la moins ambiguë de celles dont nous disposons, prenne place à l'intérieur du récit de la batai-

50 Les corrections proposées par Dobson, 1996: p. 234-235 sont peu convaincantes. Il identifie dix blocs, désignés A-J, répartis en deux rangées nord-sud de doubles baraques et rapportés aux dix cohortes de la légion. Mais la précision de cette restitution est affaiblie par les réserves de l'auteur lui-même, qui finit par reconnaître l'insuffisance des données: «the layout of the camp, on which any discussion of its garrison is based, is itself based on much conjecture.» Cependant cette prudence disparaît par la suite lorsque l'auteur d'utilise son interprétation comme argument pour appuyer ses restitutions de Renieblas VI ou de Molino.

51 L'interprétation des très rares vestiges du plan interne de Molino est assez révélatrice des limites de la méthode suivie par Dobson. Il entreprend en effet (p. 279-282) de corriger arbitrairement la restitution déjà arbitraire de Schulten, en transformant en un bâtiment unique les structures A et B, attribuées par le savant allemand à deux constructions alignées, en dépit de l'absence de toute trace bâtie entre eux. Cette première conclusion sert ensuite d'argument pour restituer les structures $\mathrm{C}$ et $\mathrm{D}$, censées appartenir, sans plus de certitude, à un second hemistrigium parallèle au premier: «The northern limit of $C / D$ is uncertain, but like $A / B$ could have extended as far as the northern edge of the terrace. The location of the southern end of $C / D$ is also uncertain but was presumably similar to that of $A / B$. It is conceivable therefore that $A / B$ and $C / D$ were similar in length.» La comparaison systematique entre une série de restitutions tout aussi conjecturales forme alors le socle de la réflexion d'ensemble de l'auteur: «A degree of validity is given to this overall reconstruction of a pair of blocks at Molino by them having an overall width, which was approximately $22 \mathrm{~m}$ at the southern end, comparable to those at Castillejo and Peña Redonda.» Plus raisonnablement, Pamment, 1996: p. 110 se déclare incapable de se prononcer sur la morphologie de Molino.

52 La prudence s'impose donc au moment d'appliquer un modèle théorique pour comprendre l'organisation des rares vestiges retrouvés. La difficulté de concilier cette prudence avec la tentation de parvenir malgré tout à un résultat est bien illustré par les contradictions du discours de Dobson. Il reconnaît (p. 134) que «the layout of these may be atypical compared to castra in general and so the above discussion of the layout of camps could be irrelevant.», mais admet que les sites de Numance devaient reproduire au moins dans une certaine mesure en miniature la structure des grands camps, si bien que: «as a result, applying the general theoretical model to the siege sites has some justification.» Par conséquent, ayant accepté au départ (p. 67) la théorie de Bell, il en cherche la vérification sur le terrain. Ce faisant, il n'est pas si éloigné de la démarche de Schulten, qu'il critique pourtant sévèrement.

53 C'est sans doute le cas à Peña Redonda où toutes les constructions sont regroupées sur la seule partie plane du site, sur son côté oriental. On ne peut exclure que le recours à une morphologie différente des baraquements répondait donc au besoin d'exploiter au mieux l'espace disponible, plutôt qu'à celui de refléter une organisation tactique nouvelle. Voir à ce sujet les remarques de Pamment, 1996: p. 77. 
lle d'Ilipa, c'est-à-dire d'un affrontement en formation contre une armée carthaginoise où l'infanterie lourde africaine, et non sa composante indigène, est présentée comme l'élite des troupes.

\section{QUELLE EXCEPTION HISPANIQUE?}

Ce qui précède suffit, je crois, pour affirmer que la cohorte, dans la mesure où elle n'obéit pas à la fonctionnalité rigide que lui prête Bell, ne peut avoir pour cette raison répondu à un besoin tactique dont les caractéristiques de la guerre en Hispania ne démontrent pas clairement l'existence. Comment comprendre dès lors l'abondance, dans les sources littéraires, des occurences de la cohorte légionnaire dans les récits relatifs à la conquête de la péninsule Ibérique ? On va voir qu'il convient de nuancer sur ce point l'originalité du dossier documentaire concernant l'Hispania.

Le terme cohors apparaît fréquemment dans les sources littéraires. Le plus souvent, il désigne explicitement les contingents de socii $^{54}$. Il est plus rarement possible de l'associer aux légions romaines. Appliqué en ce sens pour les premiers siècles de la République, il est à juste titre suspect d'anachronisme ${ }^{55}$. En outre, le manque de rigueur des auteurs anciens les conduit à appeler génériquement cohors différents types d'unités, y compris des formations barbares ${ }^{56}$. L'ambiguïté est encore plus patente dans la littérature de langue grecque où $\langle\sigma \pi \varepsilon$ ì $\rho \alpha »$ traduit «manipule» chez Polybe, mais «cohorte» chez les auteurs d'époque impériale, comme Plutarque, Dion Cassius ou Denys d'Halicarnasse. Bell a montré de façon convaincante qu'Appien utilise vraisemblablement, en fonction de ses sources, le terme dans ses deux acceptions, recourant à $« \tau \dot{\alpha} \xi \varepsilon \imath$ ls lorsqu'il évoque pour le $\mathrm{II}^{\mathrm{e}}$ siècle une unité manifestement supérieure au manipule, mais inférieure à la légion ${ }^{57}$. Cette confusion terminologique a conduit l'historiographie à adopter depuis Marquardt une attitude hypercritique, notamment envers l'ensemble des mentions du texte livien: dans cette perspective, le caractère irréfutable du témoignage polybien pour la Seconde Guerre Punique pouvait seulement être considéré comme une exception, reflétant tout au plus l'usage de la cohorte comme un expédient temporaire ${ }^{58}$.

L'apport du travail de Bell a été de montrer que ce rejet global est injustifié. Sachant que Polybe atteste l'existence de la cohorte en 206 en Hispania, plusieurs des mentions de TiteLive relatives à ce même théâtre d'opération ont été reconsidérées par lui sur cette base: il en revendique seize, sans insister melheureusement sur le détail de ses critères de sélection, hormis le contexte hispanique de ces passages ${ }^{59}$. Sur ce total, quelques-unes de ces propositions semblent raisonnables, à commencer par l'interprétation du récit de la bataille d'Ilipa, compatible avec l'information polybienne: la précision «romano milite cornibus firmatis» laisse à mon avis peu de doutes quant à la présence de cohortes romaines parmi les unités alliées composant les ailes («Ita deductis cornibus cum ternis peditum cohortibus ternisque

54 C'est son sens le plus fréquent chez Tite-Live, avec une quarantaine d'occurrences.

55 J'ai ainsi relevé vingt-sept mentions de la cohorte en contexte légionnaire dans la première décade de Tite-Live. Mais la nature des troupes dont il est question n'est pas toujours claire cependant. Le contexte montre que, lorsque le terme est manifestement rapporté à des soldats romains, il sert commodément à l'auteur pour désigner un contingent inférieur à une légion entière. Trois passages de Frontin appartiennent également à cette catégorie (Front., Strat., 1, 6, 1; $2,12,1 ; 4,1,29)$

56 Ce cas de figure représente un nombre considérable des mentions liviennes (trente-deux au total), ce qui s'explique sans doute par l'utilisation de sources grecques, pour la terminologie desquelles il fallait trouver un équivalent. Voir aussi Front., Strat., 1, 6, 3; 4, 1, 7.

57 Bell, 1965: p. 406.

58 Polybe, 11, 23, 1 et 11,33, 1.

59 Bell, 1965: p. 405: il évoque dix-sept références, mais n'en cite que seize en note. 
equitum turmis ad hoc uelitibus citado gradu in hostem ducebant sequentibus in obliquum aliis.») ${ }^{60}$. De même, rien ne s'oppose à ce que les cohortes mentionnées à propos de la mutinerie du Sucro la même année aient pu être en partie légionnaires ${ }^{61}$. En effet, je ne crois pas que les huit mille mutins aient été principalement des socii: dans ce cas, on ne comprendrait pas les reproches méprisants que Scipion leur adressa, dans son allocution à Carthagène, pour avoir choisi pour chefs Atrius d'Ombrie et Albius de Cales ${ }^{62}$. Le contexte dans lequel apparaissent les occurrences de la cohorte à cette occasion est en outre celui d'un fractionnement de l'armée en déplacement et non d'une division administrative («illa dubitatio erat, singulae ne cohortes an uniuersi ad stipendium petendum irent.»). Enfin, l'affrontement contre les Ilergètes, toujours en 206, suggère que le regroupement tactique en cohorte pouvait bel et bien répondre à un besoin ponctuel sur le champ de bataille: «quattuor cohortes in fronte statuit quia latius pandere aciem non poterat.» ${ }^{63}$. Dans ces conditions, on conviendra avec Bell que la référence à deux reprises à une cohors romana, pour 211 et 195, est plausible et que toutes les mentions liviennes acceptables ne peuvent donc s'appliquer uniformément aux seuls alliés ${ }^{64}$. Ce constat laisse par conséquent la possibilité d'interpréter en ce sens certaines mentions ambiguës, sans permettre toutefois de tirer des conclusions trop fermes dans tous les cas ${ }^{65}$.

La cohérence du dossier relatif à l'Hispania n'est cependant pas aussi stricte que l'affirme l'auteur. Certains des textes qu'il retient doivent être à mon sens écartés, faute d'éléments suffisamment solides. Frontin, Strat., 2, 6, 2, pour 211, ne fait ainsi aucune allusion à la cohorte ${ }^{66}$. La mention explicite d'une cohors romana à propos de Marcius en 211 ne suffit pas à prouver qu'il s'agit du même épisode ${ }^{67}$. De même, une anecdote récurrente relative à Q. Metellus Macedonicus en 143 se réfère à cinq cohortes $^{68}$. A lui seul, le contexte ne permet pas d'exclure qu'il s'agisse de socii. Il convient en effet de ne pas considérer que, du moment que l'existence de la cohorte légionnaire paraît attestée en Hispania, toute mention de cohorte dans les sources pour ce théâtre d'opération serait automatiquement légionnaire. Contrairement à ce que prétend Bell, le manipule y demeure présent, dans le récit li-

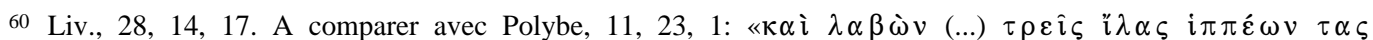

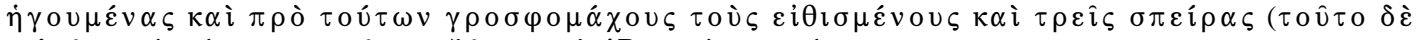

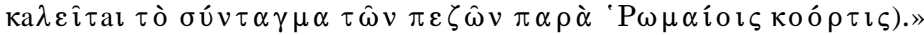

61 Liv., 28, 25, 15.

62 Liv., 28, 27, 14-15 et $28,4$.

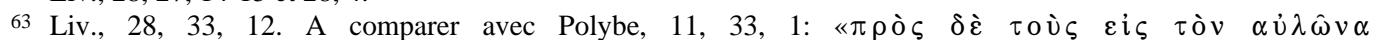

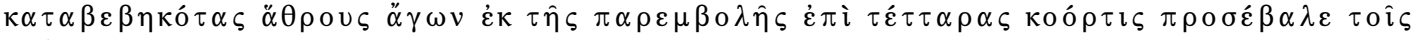
$\pi \varepsilon \zeta o \hat{\imath} \zeta . »$

64 Liv., 25, 39, 2 (pour 211) et 34, 20, 3 (pour 195). Dans le premier cas, il est préférable de demeurer toutefois prudent, avant de le considérer comme la «earliest certain reference (...) to a cohors Romana». Rappelons que le détail du récit livien des exploits de Lucius Marcius en 211 ne jouit pas d'un grand crédit dans l'historiographie relative à la Seconde Guerre Punique, qui a eu tendance à y voir une invention annalistique destinée à compenser le désastre des Scipions: voir à ce sujet les remarques de Scullard, 1929: 53 et Lazenby, 1978: p. 131. Cette réserve ne suffit pas à enlever toute fiabilité à l'information transmise par Tite-Live à propos de la cohorte. Mais elle incite à ne pas lui accorder un crédit excessif afin de fixer un terminus ante quem à sa création. Bell, 1965: p. 415 estime en effet que, puisque Tite-Live ne fait état d'aucune innovation en la matière de la part de Marcius, il faut alors attribuer celle-ci aux premiers Scipions, dont les activités sont moins bien documentées. Le raisonnement est assez fragile.

65 On ne peut ainsi exclure que les «cohortes duas» de la bataille de Baecula (Liv., 27, 18, 10) ou celles qui apparaissent pour 206 en Liv., 28, 13, 8 et 28, 23, 8 soient effectivement, faute de précision contraire, des cohortes légionnaires. Mais il est sans doute excessif de les tenir sans conteste comme telles, comme le fait Bell.

66 Il est inclus par Bell dans le dossier sur la seule foi de la formule «laxatis manipulis», dont on a vu supra (p. 148-149) qu'elle ne saurait en aucun cas, contrairement à ce que prétend l'auteur, impliquer une formation en cohortes.

67 Liv., 25, 39, 2.

68 Front., Strat., 4, 1, 23: «Q. Metellus Macedonicus in Hispania quinque cohortes quae hostibus cesserant testamentum facere iussas, ad locum reciperandum remisit, minatus non nisi post uictoriam receptum iri.» Cet exemplum est connu également par Val. Max., 2, 7, 10 et Vell. Pat., 2, 5, dans les récits desquels figurent également le terme cohors. 
vien, comme unité tactique ${ }^{69}$. On en a trace au moins pour 206, c'est-à-dire pour une année où la cohorte, de l'aveu même de Bell, est le plus explicitement attestée ${ }^{70}$. Il paraît donc impossible d'envisager une substitution sur ce plan, d'autant plus que chez Polybe, la référence au manipule demeure dominante, y compris pour l'Hispania ${ }^{71}$.

En outre, l'extrême pauvreté des mentions du manipule en contexte tactique pour la péninsule Ibérique chez Tite-Live n'a rien de particulier, car il en va de même pour les autres théàtres d'opération pourtant bien couverts par son récit. On ne compte ainsi pas plus de trois références pour la Seconde Guerre Punique: une pour l'armée de Campanie en 209, une pour celle du Bruttium en 207 et une pour l'armée de Scipion à Zama en 20272. Pour la première moitié du $\mathrm{II}^{\mathrm{e}}$ siècle, on dispose seulement de deux mentions: une pour 178, à propos de la campagne du consul Manlius en Histrie et une autre pour 171 relative au siège d'Haliarte en Béotie par le préteur Lucretius ${ }^{73}$. Cela fait bien peu pour conclure, comme le fait Bell, que Tite-Live évoque la cohorte pour l'Hispania et le manipule ailleurs ${ }^{74}$. D'autant plus que l'historien augustéen est loin de réserver le terme «cohors Romana» ou «cohors legionariae» à la péninsule Ibérique: on ne trouve pas moins de quatre références -soit deux de plus que pour les campagnes hispaniques-, toutes relatives aux affaires de Grèce. La première concerne l'aide apportée à l'automne 208 par Sulpicius Galba aux Etoliens contre Philippe V: «ipse rex cum equitatu in cohortem Romanam incurrit.» ${ }^{75}$; la seconde est relative aux opérations de 199, toujours contre le souverain macédonien: «nunc auiditate caedis intemperantius secuti in praegressas cum tribunis militum cohortes Romanas incidere.» ${ }^{76}$; la troisième se situe au cours du siège d'Elatia en 198: «simul et cohors Romana per apertum recenti strage iter inuasit.» ${ }^{77}$; la dernière est datée de l'affrontement entre Nabis et Flamininus en 195: «primae legionariae cohortes ibant, leuis armatura et equites cogebant.» ${ }^{78}$ Certes, rien n'assure que Tite-Live emploie toujours l'expression dans son sens technique. Mais, il n'y a pas a priori davantage de raisons d'écarter ces témoignages plutôt que ceux concernant la péninsule Ibérique. Ils contredisent en tout cas sans conteste l'idée selon laquelle les sources suggèrent clairement que les guerres en Orient privilégiaient tactiquement le manipule sur la cohorte, à l'inverse de celles d'Occident. Peut-on alors envisager une autre explication à l'apparition dans la documentation d'une cohorte légionnaire en Hispania dès le début de la conquête?

En fait, si l'on s'en tient strictement aux textes où les cohortes légionnaires sont probables ou vraisemblables, il est possible de les répartir, pour l'Hispania, essentiellement en

69 Bell, 1965: p. 405: «By contrast, Livy at no time mentions the maniple in Spain.»

70 Liv., 28, 19, 9: «scalas electis per manipulos uiris diuidunt.». Les mentions de «triariorum» en 207 (Liv., 28, 3, 14) et de «principes hastatosque» pour 195 (Liv., 34, 15, 16) constituent également des indices de la continuité de l'emploi tactique du manipule, trop rapidement rejetées par Bell, 1965: p. 404. Quoi qu'il en soit, il est certain que le manipule est resté l'unité administrative de base: pour 211, Tite-Live désigne normalement les légionnaires comme manipulares: «neque sedari lamentatio poterat excitantibus centurionibus manipulares» (Liv., 25, 37, 10). L'habitude en est restée jusqu'à la fin de la République, comme le prouve Cic., Phil., 5, 5, mais dans un contexte extra-péninsulaire.

71 Polybe, 10, 15, 9; 10, 35, 5; 11, 22, 10; 11, 23, 6. Ce fait est reconnu par Bell, 1965: p. 414, mais il l'explique par le fait que Polybe a sous-estimé l'importance de la nouvelle division tactique, à la fois pour des raisons personnelles (sa formation grecque le rendait incapable de prendre la mesure de la guerilla indigène) et pour des raisons circonstancielles (il se rendit en Espagne avec Lucullus en 151-150, lequel aurait mené une stratégie timide de concentration de ses forces, ne donnant pas l'occasion à Polybe d'observer la cohorte). Aucun de ces deux arguments ne peut être sérieusement soutenu.

72 Pour 209: Liv., 27, 13, 7-9 et 14, 10; pour 207: Liv., 27, 41, 6 et 27, 42, 3; pour 202: Liv., 30, 33, 1; 30, 33, 3 et 30, 33, 15. Hors contexte tactique, le manipule est cité deux fois, pour désigner les combattants, en 215 (Liv., 23 , 42,9 : «manipulatim») et en 212 (Liv., 25, 14, 7: «manipulares sui»).

73 Pour 178: Liv., 41, 1, 5 et 41, 2, 3; pour 171: 42, 63, 5.

74 Bell, 1965: p. 409: «Livy speaks consistently of the cohort in Spain and the maniple elsewhere.»

75 Liv., 27, 32, 4.

76 Liv., 31, 37, 5.

77 Liv., 32, 24, 3.

78 Liv., 34, 28, 7. 
deux groupes: lorsqu'elles ne concernent pas les campagnes de Scipion dans la seconde moitié de la Seconde Guerre Punique (et notamment pour l'année 206), elles se réfèrent toutes à celle de Caton en $195^{79}$. On peut dès lors se demander dans quelle mesure cette répartition ne reflète pas d'abord la nature des sources utilisées par Polybe et Tite-Live à ces moments de leur récit ${ }^{80}$. On sait en effet que Polybe s'est appuyé sur une documentation de première main importante, dans laquelle les témoignages oraux des acteurs, en particulier celui de Laelius, ont tenu une part essentielle ${ }^{81}$. Quant au livre XXXIV de Tite-Live, consacré en grande partie à la campagne de Caton, il occupe une place particulière dans l'ensemble de l'œuvre: on y trouve en effet un foisonnement de détails et une précision inhabituelle dans les termes techniques ${ }^{82}$. Cette caractéristique s'explique sans doute par le fait que Tite-Live a puisé dans le récit que le consul avait lui-même donné de sa campagne de Citérieure ${ }^{83}$. Il est significatif dès lors que les deux seuls passages d'Appien et de Plutarque, pour lesquels Bell estime que le vocabulaire employé correspond peut-être à une description de cohorte pour le $\mathrm{II}^{\mathrm{e}}$ siècle, se rapportent précisément aux opérations de Caton autour d'Emporion ${ }^{84}$. Par conséquent, l'éventuelle surreprésentation de la cohorte dans le contexte de l'Hispania pourrait tenir au hasard de la documentation dont la précision inhabituelle éclaire un aspect ordinairement ignoré dans son détail par les sources.

En effet, l'ensemble des occurrences potentielles de la cohorte légionnaire en péninsule Ibérique a trait à des manoeuvres ponctuelles, effectuées au cours du combat, et pour lesquelles la petite taille du manipule est inadaptée: mouvement tournant complexe (Polybe, 11, 23, 1; Liv., 28, 14, 17; Liv., 34, 14, 10), adaptation du dispositif de bataille au terrain (Polybe, 11, 33, 1; Liv., 28, 33, 12), réserve (Liv., 34, 15, 1; App., Ib., 40), détachement pour une embuscade (Liv., 25, 39, 1; Liv., 34, 20, 5), pour tenir un poste avancé (stationes) (Liv., 27, 18, 10; Liv., $28,13,8$ ) ou pour provoquer l'ennemi (Liv., 34, 14, 1; Liv., 34, 19, 9). Il semble également que des garnisons aient été formées de plusieurs cohortes (Liv., 28, 23, 8; Liv., 28, 25, 15) et qu'une éventuelle division de l'armée en campagne se soit faite sur cette base (Liv., 34, 12, 6; Liv., 34, 19, 10; Liv., 34, 20, 3). Cet emploi purement tactique explique alors pourquoi la cohorte est citée par Polybe à l'occasion de deux récits de bataille, mais pas dans son livre VI qui décrit l'agmen et non l'acies dont la présentation ultérieure, annoncée, est perdue ${ }^{85}$.

Cette formation légionnaire temporaire coexistait cependant avec une structure alliée permanente portant le même nom, source de confusion, chez les auteurs anciens comme chez les historiens modernes. Ainsi, un fragment des Orationes de Caton mentionne bien une cohors: «interea unamquamque turmam manipulum cohortem temptabam quid facere possent; proeliis leuibus spectabam cuiusmodi quisque esset; si quis strenue fecerat, donabam hones-

79 Sur les réserves relatives à la cohors Romana de Marcius en 211, voir supra, n. 64 .

80 Bell, 1965: p. 405 se contente de citer Klotz, dans FHA, III, p. 52, qui fait remonter l'essentiel des passages de Tite-Live à Coelius Antipater, Claudius Quadrigarius et Valerius Antias. Le premier, qui écrit dans les années 120, serait la source la plus utilisée par l'historien augustéen pour l'Hispania. De même, Rawson, 1971: p. 18 estime que Tite-Live ne mentionne que la cohorte pour l'Occident, car, contrairement à son récit pour l'Orient où il s'appuie essentiellement sur Polybe, il a privilégié, pour l'autre partie de l'Empire, les annalistes, moins fiables. Elle reconnaît toutefois la qualité supérieure de l'information de Coelius. Vrai dans l'ensemble, cette sélection de l'information par Tite-Live ne doit pas être systématisée, en particulier pour la Seconde Guerre Punique, où il est clair que le récit des campagnes hispaniques doit beaucoup à Polybe.

81 Nul doute qu'il a tenu du lieutenant de Scipion de nombreux «petits faits vrais» concernant les opérations de celui-ci en péninsule Ibérique. cf., Polybe, 10, 3, 2.

82 Le récit des opérations de 195 fait état d'un vocabulaire rarement employé par ailleurs chez Tite-Live. cf. Walsh, 1966: p. 163; Martínez Gázquez, 1974: p. 65; Astin, 1978: p. 28.

83 Cette hypothèse est la plus probable, selon la majorité des spécialistes: Janzer, 1936: p. 15-16; Fraccaro, 1956: p. 193-197; Martínez Gázquez, 1974: p. 69-70; Astin, 1978: p. 302-307. Il est cependant impossible de déterminer si TiteLive a lu Caton directement ou bien par l'intermédiaire d'une autre source.

84 Plut., Cato, 11; App., Ib., 40.

85 Cet argument a déjà été suggéré, à juste titre, par Rawson, 1971: p. 19. 
te, ut alii idem uellent facere, atque in contione uerbis multis laudabam.» ${ }^{86}$ Mais le contexte implique que le consul de 195 évoque ici ses contingents de socii, car il s'agit à mon sens d'une énumération de ses trois catégories de troupes: cavalerie (turma), infanterie légionnaire (manipulus) et infanterie alliée (cohors) ${ }^{87}$. Il est peu probable qu'à deux unités administratives, il ajoute, sur le même plan, une formation tactique: il faut donc qu'il s'agisse également d'une désignation administrative. Ce fragment est malheureusement le seul, attribuable à Caton, nommant la cohorte: toutefois, le fait qu'il associe manifestement celle-ci aux socii n'interdit pas que, par ailleurs, l'orateur ait pu employer le terme dans un sens différent ${ }^{88}$. Il n'est pas impossible alors que le récit livien, peu attaché on le sait à la rigueur technique, n'ait pas toujours clairement marqué ou compris la différence. Ceci expliquerait la difficulté à interpréter de manière assurée certains passages relatifs à la campagne de 195, par exemple lors de la bataille d'Emporion, où il est difficile de déterminer si les détachements sont alliés ou légionnaires ${ }^{89}$. De même, à propos du simulacre de préparatifs de départ auquel se livra le consul devant les ambassadeurs ilergètes, peu après son arrivée, la nature des troupes dont il est question n'est pas précisée: «denunciari militum parti tertiae ex omnibus cohortibus iubet ut cibum quem in naues imponant mature coquant. $\gg^{90} \mathrm{Il}$ est très improbable que les instructions de Caton n'aient été destinées qu'aux seuls socii. Il faut donc supposer ou bien une erreur de Tite-Live ou bien que nous nous trouvons ici dans un cas similaire à ce que suggère par ailleurs le récit, où la cohorte servait à diviser les légions en corps expéditionnaires ${ }^{91}$. On sait en effet que, par ce stratagème, le consul voulait précisément éviter d'avoir à affaiblir son armée («minuere exercitum»), tout en contentant ses alliés indigènes ${ }^{92}$.

Ces incertitudes compliquent l'analyse, mais il est tentant d'en conclure que l'apparition de la cohorte dans les textes relatifs à la période antérieure au $\mathrm{I}^{\mathrm{er}}$ siècle ne peut être restreinte à une explication unique: l'anachronisme flagrant cotoie la référence aux alliés, sans exclure la mention d'une authentique formation tactique légionnaire. La cohérence interne aux sources ne doit pas être surestimée. D'un livre à l'autre, voire à l'intérieur d'un même livre, la présence et la signification du terme peuvent donc varier selon le contexte, mais surtout en fonction de la documentation utilisée par l'auteur. Souvent interprétée comme le reflet de l'infériorité de la tradition annalistique, prédominante dans les affaires d'Occident, la concentration des mentions d'une cohorte pré-marienne pour l'Hispania pourrait au contraire procéder en partie du recours, très ponctuel et très inégal cependant, à une information de première main plus précise que d'ordinaire. Sur le plan tactique, la spécificité des armées romaines combattant en péninsule Ibérique aux $\mathrm{III}^{\mathrm{e}}$ et $\mathrm{II}^{\mathrm{e}}$ siècles n'est donc qu'apparente.

\section{CONCLUSION}

Les arguments parfois avancés pour mettre l'apparition et le développement de la cohorte légionnaire en relation avec les guerres menées par Rome en péninsule Ibérique aux $\mathrm{III}^{\mathrm{e}}$ et $\mathrm{II}^{\mathrm{e}}$ siècles manquent de fondements suffisamment solides. Les traditions guerrières hispaniques,

\footnotetext{
86 ORF, Cato, frgt 35. Martínez Gázquez, 1974: p. 120 a rattaché de façon plausible le contenu de ce fragment aux préparatifs de la bataille d'Emporion relatés en Liv., 34, 13 et App., Ib., 39.

87 Rawson, 1971: p. 19, n. 20 et Dobson, 1996: p. 67 admettent donc à tort, selon moi, que la cohorte dont il est question est légionnaire.

88 Peut-être en précisant «cohortes romanae», ce dont aurait alors une trace en Liv., 34, 20, 3.

89 Liv., 34, 14, 1 («sub ipsum uallum très cohortes mittit») et 34, 14, 10 («duas cohortes delectas ab dextro latere hostium circumduci iubet») ne sont pas exempts d'ambiguïté. Fraccaro, 1975: p. 139, n. 16, préfère ainsi attribuer le premier passage aux socii.

90 Liv., 34, 12, 6.

91 Voir Liv., 34, 19, 10 et 34, 20, 3.

92 Liv., 34, 12, 1.
} 
pas plus que celles des Cimbres ou des Teutons un siècle plus tard, ne présentaient la spécificité qu'on leur prête parfois. Ainsi, loin de trouver son origine dans les contraintes militaires propres à l'Hispania, la cohorte semble au contraire avoir constamment coexisté avec le manipule, dès la Seconde Guerre Punique au moins, sur l'ensemble des théâtres d'opération de la conquête. Cet aspect de l'exercitus républicain est très mal documenté. Mais l'extrême faiblesse des occurences fiables de la cohorte dans nos sources ne permet pas de le réfuter: il en va de même en ce qui concerne le manipule, très peu mentionné dans les récits même des campagnes militaires. Il est évident par conséquent que l'argument ex silentio est insuffisant. C'est de ce point de vue que le dossier relatif à la péninsule Ibérique présente sa véritable originalité. En effet, il permet peut-être de faire remonter certaines mentions de la cohorte légionnaire à des témoignages, comme ceux de Laelius ou de Caton, faisant place à des détails techniques ordinairement exclus du discours des historiens anciens. Ce faisant, il nous offre l'occasion de saisir, même imparfaitement, le contexte dans lequel cette unité tactique était ordinairement employée à l'époque. Circonscrite à des manœuvres précises sur le champ de bataille ou à des détachements ponctuels, comme les garnisons, la cohorte était finalement une manière alternative d'organiser et d'utiliser les manipules et non une unité en soi. Un écho s'en retrouve, ce n'est pas un hasard, chez Salluste, historien et homme de guerre, à propos de la guerre de Jugurtha: en 107, la légion manipulaire de Metellus, attaquée par l'armée numide près du Muthul, pouvait, grâce à la cohorte, changer son ordre de bataille en dépit de la confusion du combat (Sall., Bell. Iug., 49, 6). Ce n'est que plus tard, suite à la Guerre Sociale, à l'occasion de la seule transformation institutionnelle connue par l'armée républicaine depuis la création de la légion manipulaire, que changea la signification de cette subdivision.

FRANÇOIS CADIOU

Membre de la section scientifique 2000-2001 de l'EHEH (Casa de Velázquez).

\section{BIBLIOGRAPHIE}

ADCOCK, F. E. (1940): The Roman Art of War under the Republic, Cambridge.

Astin, A. E . (1978): Cato the Censor, Oxford.

Bell, M. J. V. (1965): «Tactical Reform in the Roman Republican Army», Historia, 14: 404-422.

CONNOLLY, P. (1981): Greece and Rome at War, Londres.

CONNOlly, P. (1989): «The Roman Army in the Age of Polybius», dans HACKeTt, J. (éd.), Warfare in the Roman World, Londres: 149-168.

DELBRÜCK, H. (1975): History of the Art of War within the Framework of Political History, I: Antiquity, Westport.

Dobson, M. (1996): The Roman Camps at Numantia: a Reappraisal in the Light of a Critical Analysis of Polybius' Discourse on the Roman Army, Exeter (thèse inédite).

FracCARO, P. (1956): «Le fonti per il consolato di M. Porcio Catone», dans FraCCARO, P., Opuscula, I, Pavie: 177-226.

FracCARO, P. (1975): «L'ordinamento a coorti», dans FracCARO, P., Opuscula, IV, Pavie: 137-169.

GABBA, E. (1973): «Le origini dell' esercito professionale in Roma: i proletari e la riforma di Mario», dans Esercito e società nella tarda repubblica romana, Florence.

GARLAN, Y. (1972): La guerre dans l'Antiquité, Paris.

Goldsworthy, A. K. (1996): The Roman Army at War, 100 BC-AD 200, Oxford.

GOLDSWORTHY, A. K. (2000): Roman Warfare, Londres. 
HARMAND, J. (1967): L'armée et le soldat à Rome de 107 à 50 avant notre ère, Paris.

HildeBRANDT, H. J. (1979): «Die Römerlager von Numantia. Datierung anhand der Münzfunde», Madrider Mitteilungen, 20: 238-271.

JANZER, B. (1936): Historische Untersuchungen zu den Redenfragmenten des M. Porcius Cato, Würtzburg.

KEPPIE, L. (1984): The Making of the Roman Army. From Republic to Empire, Londres.

Kromayer, J. et VeITH, G. (1928): Heerwesen und Kriegsführung der Griechen und Römer, Münich.

LAZENBY, J. F. (1978): Hannibal's War. A Military History of the Second Punic War, Warminster.

MARÍN Y PEÑA, M. (1956): Instituciones militares romanas, Madrid.

MARQUARDT, J. (1891): De l'organisation militaire chez les Romains, Paris.

MARTínez GÁZQUEZ, J. (1974): La campaña de Catón en Hispania, Barcelone.

Masquelez, J. (1887): «Cohors», dans Daremberg, CH. et Saglio, E. (éd.), Dictionnaire des Antiquités grecques et romaines, I, 2, Paris.

NiCOLET, CL. (1977): Rome et la conquête du monde méditerranéen, I: les structures de l'Italie romaine, Paris.

Pamment Salvatore, J. (1996): Roman Republican Castrametation. A Reappraisal of Historical and Archaeological Sources, BAR 630, Oxford.

PARKER, H. M. D. (1928): The Roman Legions, Oxford.

QueSADA SANZ, F. (1996): «Las fuerzas de los antagonistas», dans Indibil i Mandoni: reis i guerrers (Lleida, 14 nov. 1996 - 5 gen. 1997), Lérida: 140-144.

QuESADA SANZ, F. (1997a): El armamento ibérico. Estudio tipológico, geográfico, funcional, social y símbolico de las armas en la Cultura Ibérica (siglos VI-I a.C.), Montagnac.

QUESADA SANZ, F. (1997b): «Gladius Hispaniensis: an archaeological view from Iberia», dans FEUGÈRE, M., L'équipement militaire et l'armement de la République (IVe $-I^{e r} S$. av. J.-C.) (ROMEC $X$ - 26-28 sept. 1996), publié dans Journal of Roman Military Equipment Studies, 8: 251-270.

RAmBAud, M. (1966): L'art de la déformation historique dans les commentaires de César, Paris.

RAWSON, E. (1971): «The Literary Sources for the Pre-Marian Army», Papers of British School at Rome, 39: 13-31.

ROLDÁN HERVÁs, J. M. (1989): «La organización militar romana a mitad del siglo II: de la milicia ciudadana al ejército profesional», dans ROLDÁN HERVÁs, J. M., Ejército y sociedad en la España romana, Madrid: 17-60.

RoldÁn HERvÁs, J. M. (1996): El ejército de la república romana, Madrid.

Schulten, A. (1927): Numantia. Die Ergebnisse des Ausgrabungen, 1905-1912, III: Die Lager des Scipio, Münich.

Schulten, A. (1929): Numantia. Die Ergebnisse des Ausgrabungen, 1905-1912, IV: Die Lager bei Renieblas, Münich.

Scullard, H. H. (1929): Scipio Africanus in the Second Punic War, Cambridge.

STEINWENDER, TH. (1915): «Zur Kohortentatktik», Rheinisches Musaeum für Philologie, 70: 416-440.

Walsh, P. G. (1966): Livy: his Historical Aims and Methods, Cambridge.

WATSON, G. R. (1969): The Roman Soldier, Londres.

WheELER, E. (1979): «The Roman Legion as Phalanx», Chiron, 9: 303-318. 